\title{
Magnetic Properties and Morphology of Permalloy Prepared by the Compaction of the Powder
}

\section{Denisa Olekšáková}

Department of Applied Mathematics and Informatics, Faculty of Mechanical Engineering, Technical University in Košice, Letná 9, 04200 Košice, Slovak Republic

\begin{abstract}
Permalloy is the name of nickel-iron alloys, which after heat treatment have an initial permeability much larger than that one of pure iron and are produced usually in the form of thin sheet [1]. Therefore it is logical to attempt to prepare such material in more "bulk" form, for example in the form of a cylinder or a ring, which would be more convenient for some industrial applications. The aim of this work was to investigate the influence of the powder size on ac and dc magnetic properties of the bulk samples prepared by a hot compaction of the powder.
\end{abstract}

Keywords: Soft magnetic materials, permalloy, core losses

\section{Introduction}

Soft magnetic materials are a well-known group of materials suitable for various ac and dc electromagnetic applications [2]. Ni-based alloys have attracted considerable attention because of their good soft magnetic properties with nearto-zero magnetostriction, low core loss and high permeability, which can be used for a variety of applications, such as magnetic recording heads and electronic sensors [3]. Polycrystalline Ni-Fe materials milled in high-energy planetary ball mills also exhibit very good properties suitable for the preparation of soft magnetic bulk materials $[4,5]$. One of the ways to prepare bulk material (suitbale for some industrial applications) is the compaction of powder produced by the milling of amorphous, nanocrystalline and polycrystalline materials. The ball milling technique has been successfully used to prepare many alloys in powder form, which are suitable for compaction into a variety of shapes [6].

The aim of this work was to obtain by the analysis of the contributions of the hysteresis losses, the eddy current losses and the anomalous losses to the core losses that the fraction of all contributions strongly depends on the particle size of the powder used for preparation of the bulk material.

\section{Experimental material and methods}

The ribbon $\mathrm{Ni}_{81} \mathrm{Fe}_{19}$ (wt. \%) was produced by melt spinning and then milled using a RETSCH PM4000 planetary ball mill for 5 and 10 hours (Fig. 1).

The milling was performed under an Ar atmosphere with a speed of $180 \mathrm{rpm}$ at a ball-to-powder mass ratio of 6:1 in hardened steel vials. The handling of the powder was performed in a glove box. The samples were consolidated at $800 \mathrm{MPa}$ for 5 min at $500{ }^{\circ} \mathrm{C}$ into discs with a diameter of $10 \mathrm{~mm}$ and a height of approximately $2.5 \mathrm{~mm}$. An axial hole with a diameter of $5 \mathrm{~mm}$ was drilled into the disc to produce ring samples: 
- sample BR - a bulk sample prepared by the hot compaction of the small pieces (several $\mathrm{mm} 2$ ), prepared from the ribbon by breaking the ribbon by hand.

- sample P5 - a bulk sample prepared by the hot compaction of the powder obtained by the mechanical milling of the ribbon for $5 \mathrm{~h}$.

- sample P10 - a bulk sample prepared by the hot compaction of the powder obtained by the mechanical milling of the ribbon for $10 \mathrm{~h}$.

The structure and morphology of the samples were characterised by using an SEM (Vega 5135 MM Tescan scanning electron microscope at a $20 \mathrm{kV}$ accelerating voltage).

We have prepared coils with 35 primary turns and 50 secondary turns for ac and dc measurements. The magnetic induction $B$ is sinusoidal when measuring the frequency dependent hysteresis cycle.

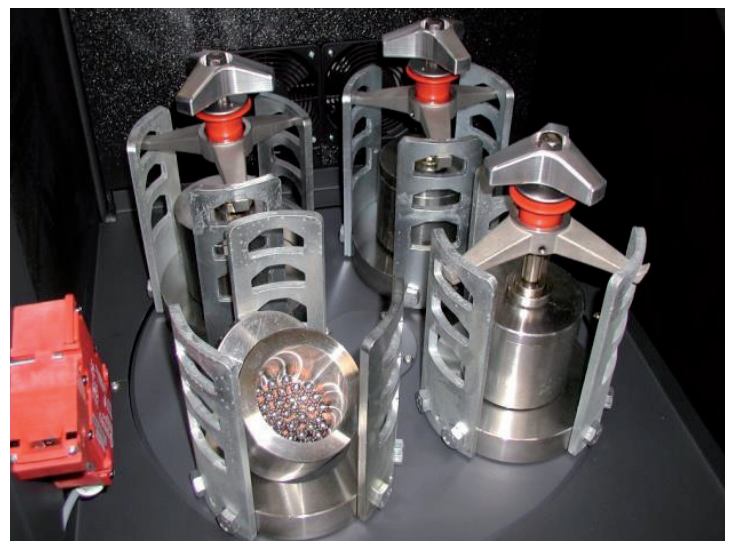

Fig. 1: Planetary ball mill RETSCH PM 4000.

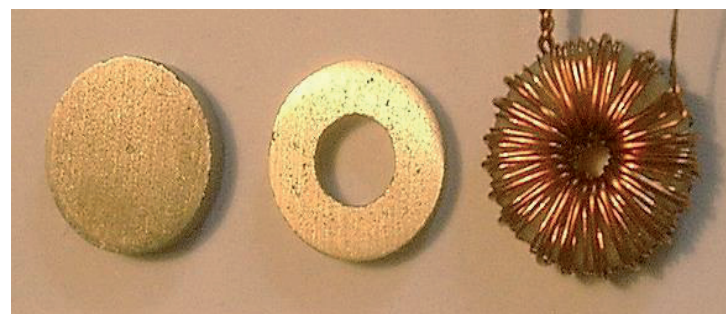

Fig. 2: Bulk samples $\mathrm{Ni}_{81} \mathrm{Fe}_{19}$ (wt.\%) prepared for ac and dc measurements.

The dc hysteresis loops at maximum flux density of $0.2 \mathrm{~T}$ were measured by a fluxmeterbased hysteresisgraph. The ac hysteresis loops in the frequency range from $0.25 \mathrm{~Hz}-50 \mathrm{~Hz}$ were measured by a fluxmeter-based hysteresisgraph with sinusoidal flux density time dependence.
The hysteresis losses Wh (dc measurements), core losses Wt (ac measurements) and coercive field were calculated directly from the measured minor hysteresis loops.

\section{Results and Discussion \\ 3.1 Structure and Morphology}

The crystalline character of all bulk samples was confirmed by X-ray diffraction with Fe filtered CoKa radiation (Philips PW 1050) with diffracted beam graphite monochromator [7, 8].

The morphology of the surface of the $\mathrm{Ni}-\mathrm{Fe}$ ribbon in as-quenched state is depicted in Fig. 3 a. Small pieces of the ribbon with area of several $\mathrm{mm} 2$ were compacted, Fig. $3 \mathrm{~d}$. The ribbon pieces were brittle enough to be crushed during compaction process and the average linear size of the pieces was reduced to $5 \mu \mathrm{m}$. The 5 hours milled ribbon, consisting of powder elements with relatively sharp edges with size of about $30 \mu \mathrm{m}$ (Fig. 3b), was precursor of the compacted bulk created by $3 \mu \mathrm{m}$ elements, Fig. 3e. The powder milled for 30 hours had average size about $10 \mu \mathrm{m}$ (Fig. 3c) and the surface of the bulk prepared from this powder is very smooth and no pores are visible, Fig. $3 f$ [4].

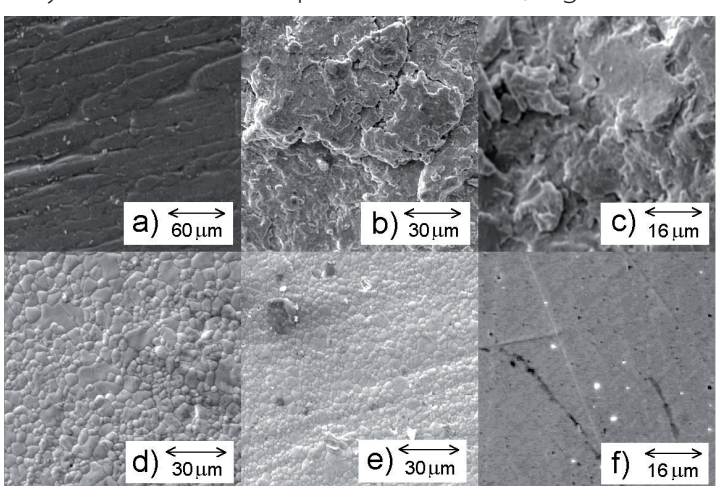

Fig. 3: The morphology a) of the Ni-Fe ribbon's surface, b) of the 5 $h$ and c) of the $30 \mathrm{~h}$ milled powder, d) of the compact prepared of broken ribbon, e) of the compact prepared from $5 \mathrm{~h}$ milled powder and f) of the compact prepared from $30 \mathrm{~h}$ milled powder [4].

\subsection{Core Losses}

The core losses $\mathrm{W}$ (in $\mathrm{J} / \mathrm{m}^{3}$ ) in the magnetic cores can be divided into three components: hysteresis loss $\mathrm{W}_{\mathrm{h}^{\prime}}$ classical eddy current losses $\mathrm{W}_{\mathrm{e}}$ and anomalous losses $\mathrm{W}_{\mathrm{a}}$ [9]

$$
W_{t}=W_{h}+W_{e}+W_{a}
$$


Hysteresis losses $\mathrm{W}_{\mathrm{h}}$ can be experimentally determined as the area of the dc hysteresis loop.

The eddy current losses We can be expressed as

$W_{e}=\frac{\pi^{2} B_{\max }^{2} d^{2}}{\beta} \sigma f=C_{e} f$

where $\mathrm{C}_{\mathrm{e}}$ is a constant in which are associated the material parameters and the parameters of the measurement conditions except for the frequency, $\mathrm{d}$ is the thickness of the sample, $\mathrm{B}_{\mathrm{m}}$ is the maximum flux density, $\mathrm{f}$ is the frequency, $\sigma$ is the conductivity, and $\beta$ is a geometrical coefficient (dependence on the width and the height of the sample).

The anomalous losses are known to be caused mainly by domain wall branching and bowing. These losses arise from the compensation of inhomogeneous internal counterfields (caused by eddy currents) by an applied magnetic field. The anomalous losses can be expressed as

$$
\begin{aligned}
& W_{a}=\frac{8.8 \sqrt{S}\left(B_{\max }\right)^{\frac{3}{2}}}{\rho} \sqrt{G \sigma V_{0}}(f)^{\frac{1}{2}}= \\
& =C_{a}(f)^{\frac{1}{2}}
\end{aligned}
$$

where $\mathrm{C}_{\mathrm{a}}$ is a constant in which are associated the material parameters and the parameters of the measurement conditions except for the frequency, $\mathrm{S}$ is the cross section of the material perpendicular to the magnetic flux, $\mathrm{G}$ and $\mathrm{V}_{0}$ are parameters that depend on the material and the magnetisation.

The dc hysteresis loops (measured at $\mathrm{B}_{\mathrm{m}}=0.2 \mathrm{~T}$ ) of all the samples are shown in Fig. 4.

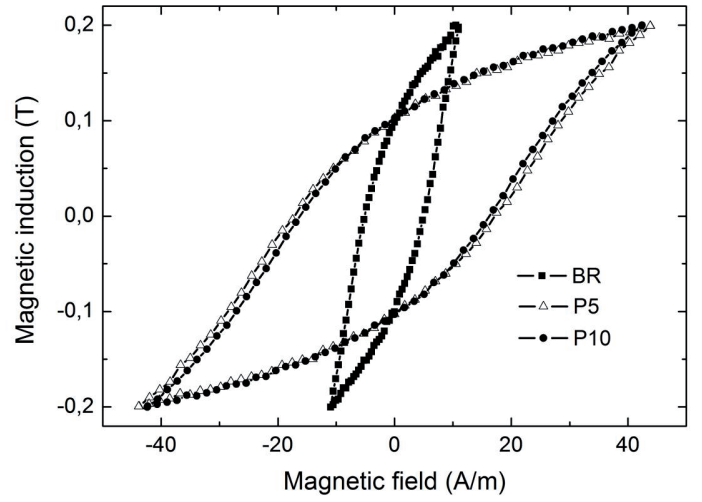

Fig. 4: The dc hysteresis loops of samples BR, P5 and P10, measured at $B_{m}=0.2 T$.
The hysteresis loop of sample BR exhibits the lowest value of the coercive field and the hysteresis losses (Tab. 1).

Table 1: Electromagnetic parameters of the samples.

\begin{tabular}{|l|l|l|l|} 
Sample & BR & P5 & P10 \\
\hline hysteresis losses $W_{h}\left(J \cdot \mathrm{m}^{-3}\right)$ & 2.8 & 10.1 & 9.7 \\
\hline coercive field $H_{c}\left(A \cdot m^{-1}\right)$ & 5.1 & 17 & 16 \\
\hline$C_{h}\left(J \cdot m^{-3}\right)$ & 2.2 & 10.5 & 9.3 \\
\hline$C_{e}\left(J \cdot m^{-3} \cdot s^{-1}\right)$ & 0.10 & 0.42 & 0.36 \\
\hline$C_{a}\left(J \cdot \mathrm{m}^{-3} \cdot \mathrm{s}^{-1 / 2}\right)$ & 1.3 & 1.1 & 1.9 \\
\hline$\beta$ & 19 & 15 & 14 \\
\hline
\end{tabular}

The higher values of the coercive field and the hysteresis losses of both samples prepared by compaction of the powder (sample P5 and sample P10) can be explained by the influence of the defects introduced during the milling process, thereby creating pinning centres for domain wall displacement.

The dc hysteresis loops (measured at maximum magnetic field of $4800 \mathrm{~A}^{-\mathrm{m}^{-1}}$ ) are shown in Fig. 5. The tendency to saturation for sample BR is significantly stronger than that for samples P5 and P10. We attribute this behaviour to defects introduced during the milling process for the samples prepared from the milled powder.

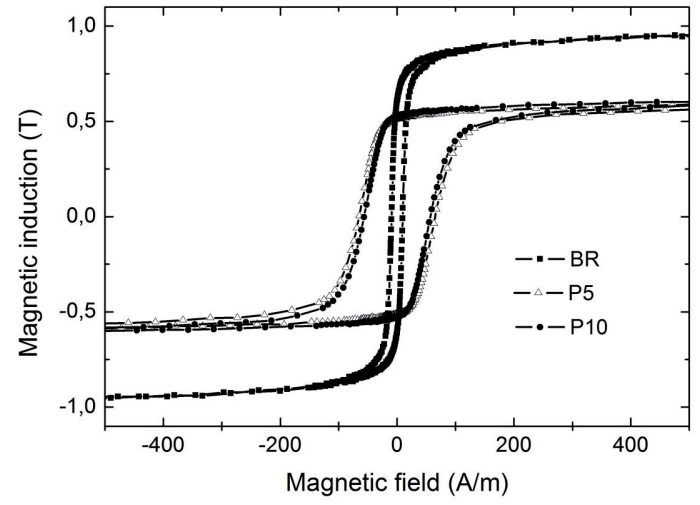

Fig. 5: The dc hysteresis loops of samples BR, P5 and P10 measured at a maximum magnetic field of $4800 \mathrm{~A} / \mathrm{m}$.

Fig. 6 shows the core losses as a function of frequency of all samples in the frequency range from dc to $50 \mathrm{~Hz}$ measured at $\mathrm{Bm}=0.2 \mathrm{~T}$. The core losses consist of all three contributions to the total 
losses: hysteresis, eddy current and anomalous. This figure shows that the frequency dependence of core losses for sample P5 and sample P10 has a crossing point at $\mathrm{f}=2.3 \mathrm{~Hz}$.

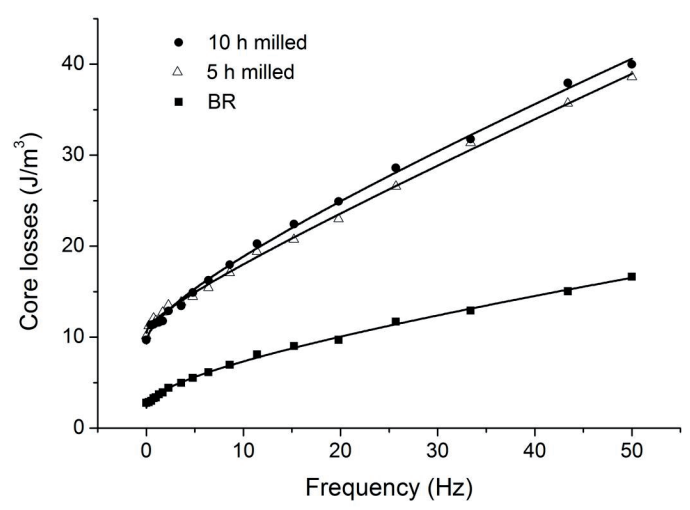

Fig. 6: The frequency dependence of the core losses measured from dc to $50 \mathrm{~Hz}$ at $\mathrm{Bm}=0.2 \mathrm{~T}$.

The frequency dependences of the core losses for all samples were analyzed according to formula based on Eq. (1), Eq. (2) and Eq. (3):

$$
W_{t}=W_{h}+W_{e}+W_{a}=C_{h}+C_{e} f+C_{a} f^{\frac{1}{2}}
$$

The frequency dependence of the core losses of sample BR exhibits the lowest values over the entire measured scale. This low loss is caused by the lowest contribution of hysteresis losses (likely caused by good movable domain walls), the lowest value of conductivity (responsible for the lowest eddy current losses) and the second lowest anomalous losses. This sample consists of the largest particles in which are likely present relatively large domain walls where carriers are present that are responsible for the non-negligible values of anomalous losses [10].

Sample P10 has the largest value of anomalous losses. The small particles of the powder fit very well to each other after compaction leading to the rise of the anomalous losses by restoring the magnetic "contact" between two particles that causes the increase of the effects. Anomalous losses can also be a reason for the undesirable large core losses at higher frequency [11]. We suppose that the smaller particles in sample P10 are responsible for the higher electrical resistivity (due to the higher number of defects in smaller particles and the higher number of electric contacts in the eddy current path) leading to the lower value of the $\mathrm{C}_{\mathrm{e}}$ coefficient in comparison with sample P5.

\section{Conclusions}

From the investigation of the dc hysteresis loss and the ac frequency dependence of the core losses of the compacted powder materials consisting of Ni81 Fe19 (wt. \%) particles of different size (5 $\mu \mathrm{m}$ - sample BR, $3 \mu \mathrm{m}$ - sample P5 and 1 $\mu \mathrm{m}$ - sample P10) we found that the lowest value of the hysteresis losses was exhibited in sample BR, which consists of the largest particles.

The most important frequency range for the detection of anomalous losses is below $5 \mathrm{~Hz}$. Omitting the frequency dependence of the core losses in this frequency range can lead to errors because of the absence of anomalous losses in the frequency range from $5 \mathrm{~Hz}$ to $50 \mathrm{~Hz}$.

Sample P5, which consists of larger particles, exhibited larger eddy current losses than sample P10.

Sample P10, which has smaller particles, exhibited the largest value of anomalous losses due to restoration of the magnetic "contact" of two neighbours particles during compaction.

Therefore, to achieve very good soft magnetic properties of compacted-powder bulk materials, it is important to study the anomalous losses as a component of the total core losses.

\section{Acknowledgments \\ This work was supported by the Scientific Grant Agency of the Ministry of Education of Slovak Republic and the Slovak Academy of Science VEGA 1/0862/12, VEGA 1/0330/15 and KEGA 072TUKE-4/2014.}

\section{References}

[1] Arnold H. D., Elmer G. W.: J. Frank Inst., 195 (1923) 621-632.

[2] H. Kronmuller, S. Parkin, Handbook of Magnetism and Advanced Magnetic Materials, Volume 4- Novel materials, Wiley, New York, 2007, ISBN: 978-0-470-02217-7

[3] P. Ripka, Journal of Magnetism and Magnetic Materials 320 (2008) 2466-2473.

[4] P. Kollár, D. Olekšáková, J. Füzer, J. Kováč, S. Roth, K. Polanski, Journal of Magnetism and Magnetic Materials 310, (2007) 2609-2611.

[5] M. M. Raja, N. Ponpandian, B. Majumdar, A. Narayanasamy, K. Chattopadhyay, Materials Science and Engineering A 
304-306 (2001) 1062-1065.

[6] J. Füzer, P. Kollár, D. Olekšáková, S. Roth, Journal of Alloys and Compounds 483 (2009) 557-559.

[7] D. Olekšáková, P. Kollár, J. Füzer, J. Bednarčík, S. Roth, Acta Physica Polonica 118 (2010) 797-799.

[8] D. Olekšáková, S. Roth, P. Kollár, J. Füzer, Journal of Magnetism and Magnetic Materials 304, (2006) e730-e732.

[9] H. Kronmuller, S. Parkin, Handbook of Magnetism and Advanced Magnetic Materials, Volume 2- Micromagnetism, Wiley, New York, 2007, 2912 p. ISBN: 978-0-470-02217-7

[10] Y. B. Kim, K. Y. Kim, IEEE Transactions on Magnetics 42(2006) 2802-2805.

[11] D. Kim, M. Ohnishi, N. Matsushita, M. Abe, IEEE Transactions on Magnetics 39 (2003) 3181-3183.

\section{Biographical notes}

RNDr. Denisa Olekšáková, PhD., (born in 1979) received PhD degree in Physics of Condensed Matter and Acoustics at the P. J. Šafárik University in Košice in 2007. At present, she works as a lecturer at the Department of Applied Mathematics and Informatics of the Faculty of Mechanical Engineering of Technical University in Košice. Her research interest is the investigation tshe structure and magnetic properties of soft magnetic materials. She is co-author of 50 scientific publications and 16 of these publications are registered in the Current Contents database, for which are more than 50 citations were recorded in the citation databases SCI and SCOPUS. 